\title{
MR Susceptibility Imaging with a Short TE (MR-SISET): A Clinically Feasible Technique to Resolve Thalamic Nuclei
}

\author{
(D) S. Chung, (D) P. Storey, (D)T.M. Shepherd, and (D).W. Lui
}

\begin{abstract}
SUMMARY: The thalamus consists of several functionally distinct nuclei, some of which serve as targets for functional neurosurgery. Visualization of such nuclei is a major challenge due to their low signal contrast on conventional imaging. We introduce MR susceptibility imaging with a short TE, leveraging susceptibility differences among thalamic nuclei, to automatically delineate 15 thalamic subregions. The technique has the potential to enable direct targeting of thalamic nuclei for functional neurosurgical guidance.
\end{abstract}

ABBREVIATIONS: MR-SISET = MR susceptibility imaging with a short TE; STh = subthalmic; CM = centromedian

$\mathbf{T}$ he thalamus plays a critical role as a central relay hub and comprises functionally specific nuclei. However, it appears relatively homogeneous in structure on conventional imaging (Fig 1A). This appearance limits the ability of physicians to accurately target specific nuclei in functional neurosurgical therapies, such as the ventral intermediate/lateral and subthalamic (STh) nuclei for the treatment of essential tremor and tremor-dominant Parkinson disease ${ }^{1}$ and the centromedian (CM) nucleus for the treatment of refractory epilepsy ${ }^{2}$ and Tourette syndrome. ${ }^{3}$ Neurosurgical outcome is critically dependent on accurate targeting; however, current clinical practice relies on indirect localization based on standard coordinates because there is no reliable, noninvasive means currently available to resolve individual nuclei. Indirect targeting is suboptimal due to anatomic variation across individuals, which may lead to inaccurate targeting with potential implications for clinical outcome. Histology-based atlases may improve accuracy ${ }^{4}$ but are subject to limitations such as the typically small number of subjects on which they are based and registration errors when mapping from atlas to patient. DTI

Received November 13, 2019; accepted after revision May 11, 2020.

From the Center for Advanced Imaging Innovation and Research (CAI2R), and Bernard and Irene Schwartz Center for Biomedical Imaging, Department of Radiology, New York University Grossman School of Medicine, New York, New York.

This work was supported by the National Institutes of Health R01 NS039135-11, R21 NS090349, P41 EB017183; and the Leon Lowenstein Foundation.

Please address correspondence to Sohae Chung, PhD, 660 1st Ave, 4th floor, NY, NY10016; e-mail: sohae.chung@nyulangone.org

\footnotetext{
- Indicates open access to non-subscribers at www.ajnr.org

Indicates article with supplemental on-line table.

Indicates article with supplemental on-line photo.

http://dx.doi.org/10.3174/ajnr.A6683
}

tractography techniques aimed at delineating thalamic nuclei are problematic when white matter tracts are difficult to track. ${ }^{5}$

The thalamus is a deep gray matter structure that contains a considerable amount of WM. In the thalamus, iron content, degree of WM myelination, ${ }^{6}$ and WM fiber orientation ${ }^{7}$ may all contribute to susceptibility differences within the structure. There are recent reports showing the potential of susceptibility imaging for delineating thalamic nuclei at $7 \mathrm{~T} ;{ }^{8,9}$ however, clinically practical protocols at $3 \mathrm{~T}$ are lacking. Here, we introduce a novel method, leveraging short TEs, termed MR susceptibility imaging with a short TE (MR-SISET), to bring out differences in contrast among thalamic nuclei and provide an automated method to delineate the intrathalamic subregions using $\mathrm{K}$ means clustering.

\section{Technique}

The study was approved by the local institutional review board, and all subjects provided informed consent. Ten healthy controls were included in the study spanning a broad age range (2365 years of age; 3 men) to assess intersubject variation due to agerelated myelination changes. MR-SISET was performed on 3T MR imaging scanners (Magnetom Skyra/Prisma [6/4 $\quad$ subjects]; Siemens) using a 3D multiecho gradient-echo sequence: FOV $=220 \times 170 \times 75 \mathrm{~mm}^{3}$, matrix $=176 \times 136 \times 60,1.25-\mathrm{mm}$ isotropic resolution, flip angle $=22^{\circ}, \mathrm{TR}=92 \mathrm{~ms}, 20$ multiple $\mathrm{TE}=$ $1.90-45.98 \mathrm{~ms}$ with echo spacing of $2.32 \mathrm{~ms}$, bandwidth $=840 \mathrm{~Hz} /$ pixel. MR-SISET maps were generated using the MEDI toolbox ${ }^{10}$ with a relatively high regularization parameter $(\lambda=2000)$ related to data fidelity and sharpness to optimize image contrast. Automatic delineation was performed using a K-means algorithm 


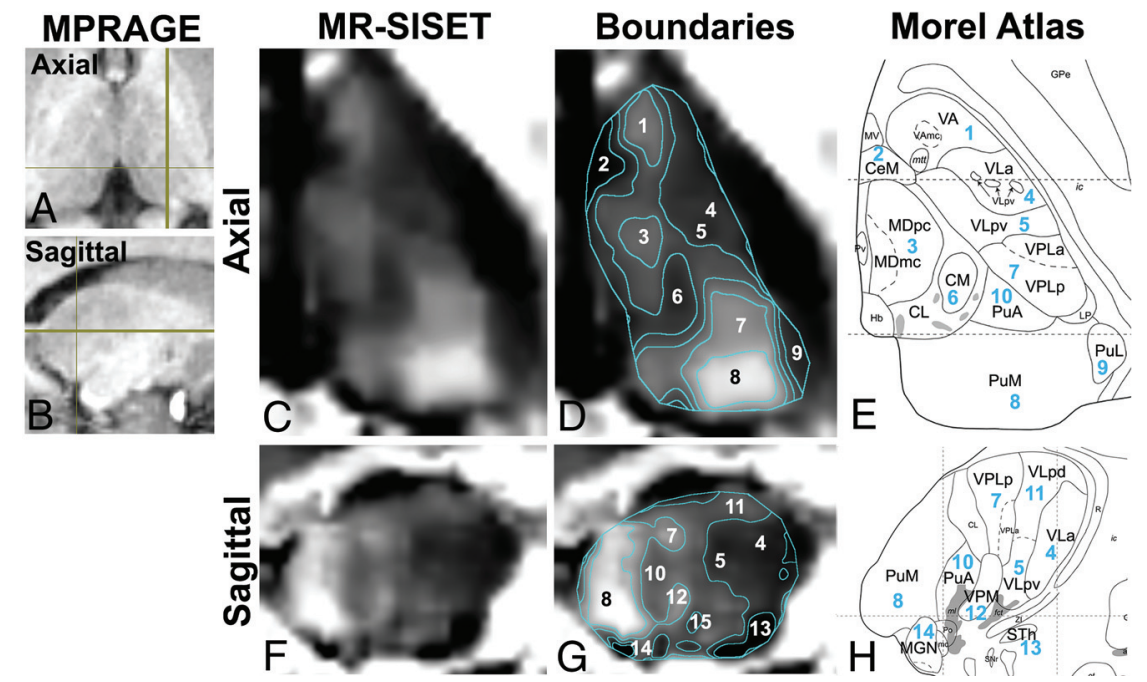

FIG 1. MPRAGE images are shown in the axial $(A)$ and sagittal planes $(B)$, indicating section location. Axial images: C, MR-SISET map. D, Boundaries of 15 thalamic nuclei derived from K-means clustering. E, Corresponding Morel atlas labels are shown. F-H, Sagittal images (1-VA, 2-CeM, 3MD, 4-VLa, 5-VLpv, 6-CM, 7-VPL, 8-PuM, 9-PuL, 10-PuA, 11-VLpd, 12-VPM, 13-STh, 14-MGN, 15-VM (not shown in $H$ ). Reprinted with permission from Morel, $2007 .^{6}$
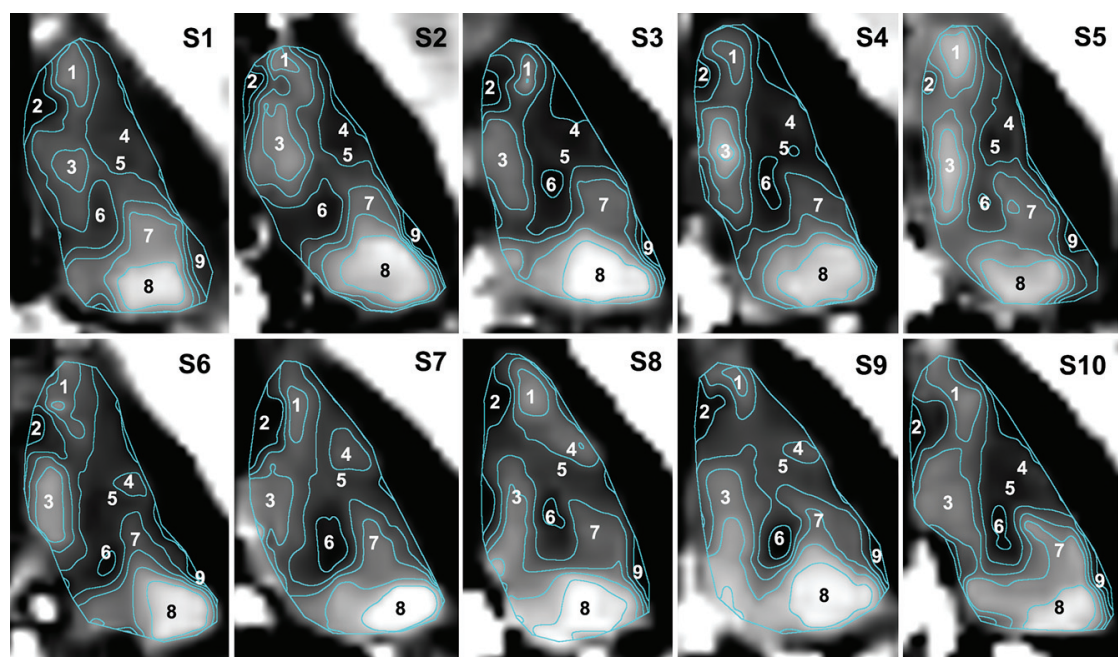

FIG 2. MR-SISET maps with corresponding boundaries for all 10 subjects, showing clear similarities between subjects with several subregions that were consistently segmented. S indicates subject. segmented and appeared to correspond to the following thalamic nuclei on the Morel atlas (Fig 2 and On-line Figure): CM (1), central medial (CeM, $0.92 \pm 0.08)$, lateral pulvinar (PuL, $0.87 \pm 0.09)$, STh (0.62 \pm 0.17 ), and ventral medial (VM $0.95 \pm 0.08)$ regions were consistently darker than their neighbors, while ventral anterior (VA, $1.32 \pm 0.09$ ), mediodorsal (MD, $1.41 \pm 0.2)$, ventral posterior lateral (VPL, $1.29 \pm 0.11$ ), ventral posterior medial (VPM, $1.29 \pm 0.13$ ), and medial pulvinar (PuM, $1.79 \pm 0.31)$ regions were brighter than their surroundings (contrast ratios are shown in parentheses). The ventral lateral anterior (VLa, $1.10 \pm 0.1)$ and ventral lateral posterior, ventral division (VLpv, $1.04 \pm 0.06$ ) regions were not easily separable. The anterior pulvinar $(\mathrm{PuA}, 1.17 \pm 0.13)$, ventral lateral posterior, dorsal division (VLpd, $1.17 \pm 0.15$ ), and medial geniculate (MGN, $1.18 \pm 0.19$ ) regions had similar contrast. The terms are defined and contrast ratios are summarized in the On-line Table.

\section{DISCUSSION}

MR-SISET delineates 15 distinct thalamic subregions at 3T, as a result of a greater sensitivity to myelin content and WM packing geometry, supported by previous works. ${ }^{11}$ A limitation of this study is the lack of ground truth. Instead, indirect comparisons have been performed against the Morel atlas, which is based on histology, though from a small number of subjects. The regions segmented using MR-SISET do appear to correspond well to nuclei delineated in the Morel atlas. Further work is warranted in patients with relevant pathology to assess the potential utility in patients

with 6 different intensity clusters (Matlab R2019b; MathWorks). Subregions were visually inspected and compared against known thalamic nuclei as delineated in the Morel atlas. ${ }^{4}$ Susceptibility contrast relative to that of the CM nucleus was calculated for each subregion.

\section{RESULTS}

MR-SISET maps showed clear internal heterogeneity with 15 distinct nuclei showing a high degree of signal contrast with neighboring nuclei (Fig 1). There were clear similarities between subjects with several subregions that were consistently undergoing functional neurosurgery.

\section{CONCLUSIONS}

MR-SISET enables subject-specific delineation of intrathalamic structures. Thus far, magnetic susceptibility-based techniques have not been widely exploited for resolving intrathalamic structures, particularly at 3T. Our preliminary results suggest that MR-SISET has the potential to aid in more personalized, direct visualization of thalamic nuclei for functional neurosurgery.

Disclosures: Sohae Chung - RELATED: Grant: National Institutes of Health R01 NS039135-11, R21 NS090349, P41 EB017183.* Pippa Storey—RELATED: Grant. National 
Institutes of Health.* Timothy M. Shepherd-UNRELATED: Stock/Stock Options: Microstructure Imaging, Comments: founder and equity for startup, no money has been paid to me or my institution. Yvonne W. Lui-RELATED: Grant: National Institutes of Health*; UNRELATED: Grants/Grants Pending: Leon Lowenstein Foundation, Department of Defense.* *Money paid to the institution.

\section{REFERENCES}

1. Benabid AL, Pollak P, Gervason C, et al. Long-term suppression of tremor by chronic stimulation of the ventral intermediate thalamic nucleus. Lancet 1991;337:403-06 CrossRef Medline

2. Son BC, Shon YM, Choi JG, et al. Clinical outcome of patients with deep brain stimulation of the centromedian thalamic nucleus for refractory epilepsy and location of the active contacts. Stereotact Funct Neurosurg 2016;94:187-97 CrossRef Medline

3. Vandewalle V, van der Linden C, Groenewegen HJ, et al. Stereotactic treatment of Gilles de la Tourette syndrome by high frequency stimulation of thalamus. Lancet 1999;353:724 CrossRef Medline

4. Morel A, Magnin M, Jeanmonod D. Multiarchitectonic and stereotactic atlas of the human thalamus. J Comp Neurol 1997;387: 588-630 CrossRef Medline

5. Sammartino F, Krishna V, King NK, et al. Tractography-based ventral intermediate nucleus targeting: novel methodology and intraoperative validation. Mov Disord 2016;31:1217-25 CrossRef
6. Morel A. Stereotactic Atlas of the Human Thalamus and Basal Ganglia. Informa Healthcare; 2007:13-53

7. Lancione M, Tosetti M, Donatelli G, et al. The impact of white matter fiber orientation in single-acquisition quantitative susceptibility mapping. NMR Biomed 2017;30:10.1002/nbm.3798 CrossRef Medline

8. Abosch A, Yacoub E, Ugurbil K, et al. An assessment of current brain targets for deep brain stimulation surgery with susceptibility-weighted imaging at 7 Tesla. Neurosurgery 2010;67:1745-56; discussion 1756 CrossRef Medline

9. Deistung A, Schafer A, Schweser F, et al. Toward in vivo histology: a comparison of quantitative susceptibility mapping (QSM) with magnitude-, phase-, and R2*-imaging at ultra-high magnetic field strength. Neuroimage 2013;65:299-314 CrossRef Medline

10. Liu T, Xu W, Spincemaille $\mathrm{P}$, et al. Accuracy of the morphology enabled dipole inversion (MEDI) algorithm for quantitative susceptibility mapping in MRI. IEEE Trans Med Imaging 2012;31: 816-24 CrossRef Medline

11. Sood S, Urriola J, Reutens D, et al. Echo time-dependent quantitative susceptibility mapping contains information on tissue properties. Magn Reson Med 2017;77:1946-58 CrossRef Medline 